\title{
Unidade piloto em regime de batelada com sistema de reatores anaeróbios + microalgas + wetlands construídos em fluxo vertical
}

\author{
Matheus Wink ${ }^{1}$ \\ William Ribeiro ${ }^{2}$ \\ Sabine Pommerehn ${ }^{2}$ \\ Júlia Fernanda Radtke \\ Elizandro Oliveira Silveira ${ }^{4}$ \\ Enio Leandro Machado
}

\section{RESUMO}

Três configurações para operar em batelada sistemas integrados que evoluíram para Reatores Anaeróbios + Microalgas + Wetlands Construídos de Fluxo Vertical (RA + MA + WCFV) foram investigadas neste trabalho, tendo as configurações as seguintes características: combinação de tanque de MA com 90L de volume útil, dotado de recirculação interna em cone de acrílico e externa com tanque de 20L, também em acrílico, sendo integrado com WCFV com tempo de detenção hidráulico (TDH) de 3 dias com a macrófita Hymenachne grumosa. A configuração do sistema RAs +MA + WCFV vem em operação nos últimos 8 meses, mostrando reduções totais de $\mathrm{N}_{-\mathrm{NH}_{4}}{ }^{+}$(com concentração inicial de $68 \mathrm{mg} \mathrm{L}^{-1}$ ), associando $50 \%$ de redução de DQO e $70 \%$ de fósforo total. Melhorias para o controle de remoção de algas residuais devem ser feitas para aplicação do WCFV, especialmente quanto a carga volumétrica, que deverá ser com até $20 \mathrm{~cm} \mathrm{dia}^{-1}$. Para continuidade da evolução foram considerados que devem ser pesquisados os seguintes aspectos: impossibilidade de operação do sistema em fluxo contínuo; drenagem do sistema de lodo não permite sua remoção completa nos Reatores Anaeróbios (RAs) e impossibilidade de recarga simultânea dos sistemas.

Palavras-chaves: Wetlands construídos . Microalgas. Efluentes urbanos.

\section{ABSTRACT}

Three configurations to operate in batch integrated systems that evolved to Anaerobic Reactors + Microalgae + Constructed Wetlands of Vertical Flow (ARs + MA + CWVF) were investigated in this work, with the following settings: MA tank combination with a volume of 90L, provided with internal recirculation and acrylic cone with external tank of 20L, also acrylic, being integrated with CWVF having hydraulic detention time (HDT) of 3 days with the macrophyte Hymenachne grumosa. The final configuration of the ARs + MA + CWVF

\footnotetext{
${ }^{1}$ Aluno do Curso de Química Industrial da Universidade de Santa Cruz do Sul - UNISC.

${ }^{2}$ Alunos do Curso de Ciências Biológicas da Universidade de Santa Cruz do Sul.

${ }^{3}$ Aluna do Curso de Química Industrial da Universidade de Santa Cruz do Sul.

${ }^{4}$ Mestrando do Programa de Pós-Graduação em Tecnologia Ambiental da Universidade de Santa Cruz do Sul.

${ }^{5}$ Professor do Departamento de Química e Física na Universidade de Santa Cruz do Sul. <enio@unisc.br>
} 
system comes into operation in the last 8 months showing total reductions of $\mathrm{N}_{-} \mathrm{NH}_{4}{ }^{+}$(with starting concentration of $68 \mathrm{mg} \mathrm{L}^{-1}$ ), involving 50\% COD reduction and $70 \%$ total phosphorus. Improvements to control removal of the residual algae must be made for application of CWVF, particularly as the volumetric load, which should be $20 \mathrm{~cm} \mathrm{day}^{-1}$. For continuity of evolution it was considered that the following should be investigated: system operation impossibility streaming; sludge drainage system does not allow complete removal in Anaerobic Reactors (RAs) and impossibility of simultaneous recharging systems.

Keywords: Constructed wetlands . Microalgae . Urban wastewater.

\section{INTRODUÇÃO}

O uso de Wetlands Construídos (WCs) utilizando macrófitas está integrado com as potencialidades de saneamento rural de baixo custo, proporcionando reuso de águas residuárias, recuperação de nutrientes, além do potencial para geração de energia. Ainda assim, podem-se destacar aspectos favoráveis, como o custo de implantação, a produção de biomassa que pode ser utilizada na alimentação animal e a eficiência de melhoria dos parâmetros que caracterizam os recursos hídricos (PHILIPPI, 2004; SEZERINO, 2006).

Apesar das inúmeras vantagens que os WCs podem apresentar esta tecnologia ainda carece de pesquisas para efetiva implantação no Brasil, mas vem ganhando espaço na área da tecnologia ambiental, principalmente em pesquisas de diversas universidades (VAN KAICK, 2002; ZERWES, 2013; SILVEIRA, 2010).

Desde 2013, por iniciativa do grupo de Estudos de Saneamento Descentralizado (GESAD) da Universidade Federal de Santa Catarina (UFSC), vem sendo organizado o simpósio Brasileiro sobre Wetlands Construídos, com realizações na própria UFSC e recentemente na Universidade Tecnológica Federal do Paraná (UTFPR) em Curitiba, fortalecendo também o envolvimento do grupo de Ciência e Tecnologia Ambiental da Universidade de Santa Cruz do Sul (UNISC).

\section{FUNDAMENTAÇÃO TEÓRICA}

Aspectos de projeto, configuração, manutenção, controle analítico e integração com outros métodos de tratamento vêm ganhando espaço para pesquisas e aplicações do saneamento descentralizado com os sistemas alagados construídos. Experiências com 
prefeituras, casas de estudantes universitários, unidades de extensão para área rural, especialmente via Emater e Embrapa ganham destaque para inclusão de macrófitas fixas, submersas e flutuantes em unidades de tratamento de esgotos (ZERWES et al., 2015; SILVEIRA, 2015).

Outro aspecto importante das pesquisas consiste no perfil de escala e operação das unidades, especialmente se for considerado o estudo de métodos. Neste caso, unidades em fluxo semicontínuo (residências) e batelada são interessantes. Fluxos contínuos envolvem normalmente contribuições de condomínios, bairros e cidades. No entanto, pesquisas feitas por Rakoczy, 2011, investigaram a biodegradação do benzeno em WCs de fluxo subsuperficial, considerando taxas de alimentação de $2 \mathrm{~L} \mathrm{dia}^{-1}$ e com tempos de detenção hidráulico de 14 dias.

Herrera-Melián et al., 2012 conceberam também um perfil de sistema de wetlands construídos em unidade de bancada. Para este caso o objetivo foi para estudos de degradação do dinitrofenol, considerando a possibilidade de integração de métodos fotoquímicos e alagados construídos. Neste caso a escala de bancada consistiu de frascos de vidro de 0,5L contendo $300 \mathrm{~g}$ de brita e até $150 \mathrm{~mL}$ de volume útil para os efluentes. A combinação de sistema Luz Solar/TiO 2 com tempo de detenção de $6 \mathrm{~h}$ e com $16 \mathrm{~h}$ para o WC citado anteriormente foi capaz de degradar totalmente $200 \mathrm{mg} \mathrm{L}^{-1}$ do composto aromático dinitrado. Já Dufresne et al., 2015, trabalharam com WCs em escala de bancada concebendo sistemas com $0,2 \mathrm{~m}^{2}$ para estudar o mecanismo de retenção de metais pesados (Ni, $\mathrm{Zn}$ e $\mathrm{Mn}$ ) com uso da planta Thypha latifólia e operando com $1,5 \mathrm{~mL} \mathrm{~min}^{-1}$ de efluentes da drenagem de minas.

Portanto, com relação aos sistemas de wetlands construídos em unidades de batelada e em unidade de bancada, há referências de confiabilidade de pesquisas, sendo adequados para estudos de processos de remediação em efluentes.

Outro aspecto das pesquisas com a fitorremediação é o da integração dos sistemas de WCs com algas. Maddox e Kingsley, 1989, investigaram o tratamento de efluentes da psicultura, os quais apresentavam algas e demais cargas poluentes em termos de $\mathrm{DQO}, \mathrm{DBO}_{5}$, nitrogênio total e fósforo total. Para este caso, o tratamento dos descartes dos tanques de criação de peixes envolveram wetlands construídos de fluxo horizontal subsuperficial (com Eleocharis dulcis), com cargas volumétricas de $0,15,0,1$ e 0,06m dia ${ }^{-1}$. As reduções de DQO e $\mathrm{DBO}_{5}$ foram de aproximadamente $75 \%$, fósforo total de $56 \%$ e nitrogênio total de $64 \%$. O controle da remoção das algas revelou redução entre 25 a $27 \%$ para tempos de detenção hidraúlico de 5 e 10 dias. LU et al., 2015, referenciam diferentes processos integrados com WCs para o tratamento dos efluentes: reatores anaeróbios + WCs; reator anaeróbio + tanque 
de aeração + WCs e Filtro Anaeróbio + WCs. Desta forma, o objetivo principal deste projeto foi desenvolver estrutura de integração de processos com microalgas e WCs de fluxo vertical, especialmente para reduzir a carga eutrofizante nos alagados construídos e, por consequência, aumentar a capacidade de carga volumétrica para a unidade de fitorremediação.

Assim sendo, aplicar unidade para o crescimento de algas associada com póstratamento por alagados construídos (com a Hymenachne grumosa, compartimentado em fluxo misto: vertical + subsuperficial - VFCW +SSFCW) para tratamento de efluentes domésticos foi o principal objetivo da pesquisa. Neste contexto, a construção e implantação de unidade para o crescimento de algas, estabelecendo parâmetros de configuração e controle para sistema em fluxo contínuo e/ou semibatelada com integração aos wetlands construídos foi desenvolvida e monitorada.

\section{METODOLOGIA}

Parte do sistema experimental da Figura 1 foi reativada, pois se tratava de instalação utilizada por uma mestranda do Programa de Pós-graduação em Gestão e Tecnologia Ambiental da Universidade de Santa Cruz do Sul - PPGTA-UNISC do ano de 2010. Este sistema está instalado na Estação de Tratamento de Esgotos (ETE), no Campus da UNISC. Neste caso foi encaminhada a configuração de Microalgas + Wetlands Construídos de Fluxo Vertical (MA + WCFV2) e Wetlands Construídos Mistos (WCFV1 + WCsFHSS). Considerase que WCsFHSS são Wetlands Construídos de Fluxo Horizontal Subsuperficial. Igualmente foi feito para os sistemas chamados de controle, a configuração de meio filtrante idêntico aos sistemas WCFV e WCsHFSS, denominados de CONTROLE (somente o meio filtrante de fluxo vertical) e CONTROLE MISTO (sequência do meio filtrante com fluxo vertical mais dois meios filtrantes de fluxos horizontais subsuperficiais).

De acordo com a Coordenação Ambiental do Campus da UNISC, o esgoto drenado para tratamento na ETE é originário dos mictórios e bacias sanitárias, sendo que os restaurantes possuem sistemas de tratamento com caixa separadora de óleos e graxas e posterior encaminhamento para a ETE UNISC.

A configuração do sistema de WCs foi constituída de 3 caixas em sequência, posicionadas sobre uma estrutura metálica em forma de degraus, estando a caixa inicial do sistema a $1,4 \mathrm{~m}$ do chão; a caixa intermediária a $75 \mathrm{~cm}$ do chão e a caixa final a $10 \mathrm{~cm}$ do chão. 
Figura 1 - Esquema geral da inserção do Sistema Reator Anaeróbio +Microalgas + Wetlands Construídos de Fluxo Vertical para as pesquisas com efluentes da ETE UNISC. RA - Reator Anaeróbio.

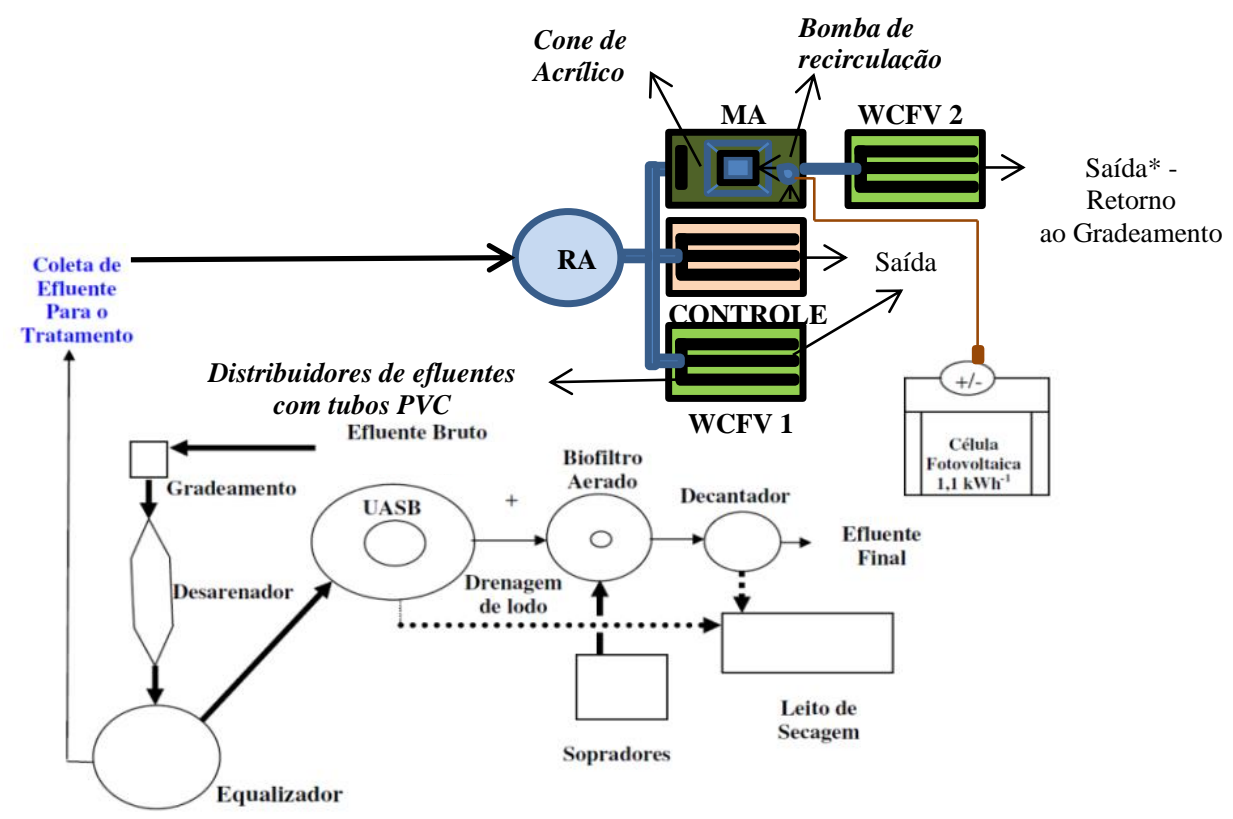

Assim sendo, no sistema da Figura 1 foram monitorados: Efluente Bruto; CONTROLE; onde as caixas foram preenchidas apenas com areia e brita, CONTROLE Final (com três caixas sequenciais); MA; WCFV1; WCFV2, WCFV1 + WCsFHSS (Wetland Final), preenchidos com areia, brita e com a macrofilas (Hymenachne grumosa).

A carga dos sistemas foi feita de 3 em 3 dias em conjunto da coleta das amostras para as análises, com efluente retirado do equalizador da ETE, carregado com 90 litros na primeira caixa de cada sistema, esses 90 litros foram retirados de um reator anaeróbio, onde o efluente bruto do equalizador permanecia por 3 dias.

Os parâmetros analisados para caracterização do efluente bruto e póstratamento foram, $\mathrm{DBO}_{5}$, nitrogênio amoniacal, fósforo total, DQO, Condutividade, $\mathrm{pH}$, cor absorciométrica e turbidez. A referência de controle das algas foi com unidades absorciométricas em $686 \mathrm{~nm}$, sendo para a cor predominante dos efluentes dos WCs foi feito o controle em 420nm. Exceto o controle de cores absorciométricas, todos os demais parâmetros foram referenciados com o APHA/AWWA, 2005. 


\section{RESULTADOS E DISCUSSÕES}

\subsection{Desenvolvimento de configurações e operação dos sistemas Microalgas e Wetlands Construídos}

Os primeiros seis meses de atividades foram direcionados para programar a ETE de escala piloto associada com a ETE em escala real da UNISC, situada no campus central. Neste caso o estudo das configurações objetivou verificar controle de vazões de alimentação, estabelecendo tempos de carga para o sistema em semibatelada, bem como o tempo de detenção hidráulico mínimo referendado nas literaturas que também trabalham em regime semelhante (HERRERA-MELIÁN et al., 2012; DUFRESNE et al., 2015).

O primeiro sistema de partida está apresentado na Figura 2, sendo ainda mantida a alimentação para fluxo horizontal em todos os WCs.

Figura 2. CONFIGURAÇÃO 1 do sistema para início do controle sistemático da integração MA + WCFV.

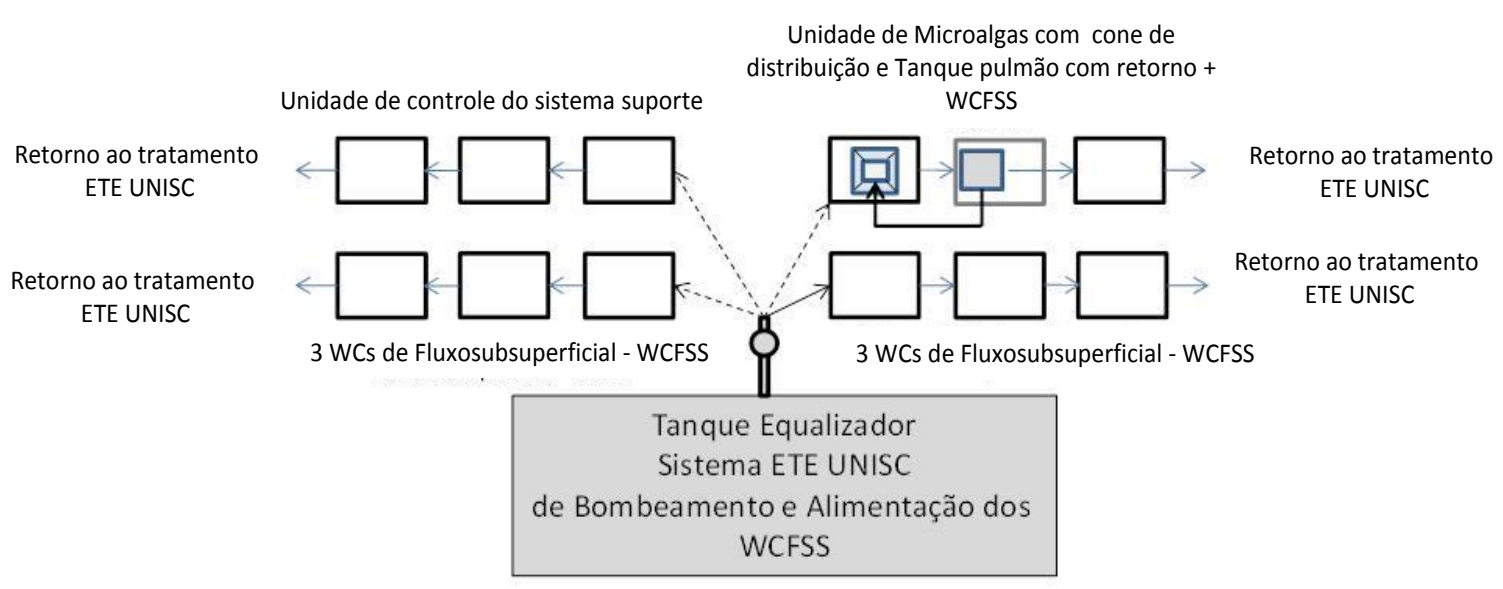

A maior dificuldade para a primeira configuração foi manter uniforme a alimentação, dadas às variações de preenchimento do tanque de equalização da ETE em escala real, pois deste ponto é retirado o efluente para a alimentação do sistema. Aqui também foi iniciada a partida do sistema de MA, composto também por alimentação com recirculação em reator tipo coluna com 20L. Este detalhamento pode ser visto na Figura 3. 
Figura 3 - Detalhe do sistema de MA com recirculação externa em reator tipo coluna com volume útil de 20 L. TQR - Tanque de Recirculação. CONFIGURAÇÃO 1.

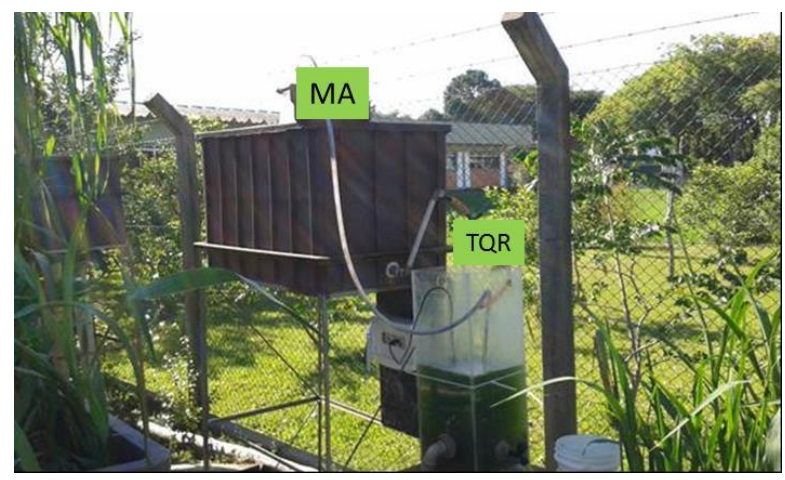

Com a configuração da Figura 3 dois pontos importantes foram observados: o desenvolvimento das microalgas (gêneros de microalgas identificados foram Desmodesmus, Scenedesmus e Chlorella), o período de sombreamento de 5 horas (configuração da ETE UNISC, um limitante sem possibilidades para resolução), os riscos de ruptura do tanque de acrílico, e o que mais foi considerado: a configuração desejada para reproduzir em um futuro, o sistema em escala real, que terá somente o cone de recirculação. O sistema da configuração 1 (Figura 3) operou 2 meses. Assim sendo, foi considerada a evolução do sistema para a Figura 4.

Figura 4 - CONFIGURAÇÃO 2 do sistema para início do controle sistemático da integração MA + WCFV (Vista superior --MA com taxa de recirculação de $0,1 \mathrm{Lmin}^{-1} \mathrm{~m}^{-2}$ ).

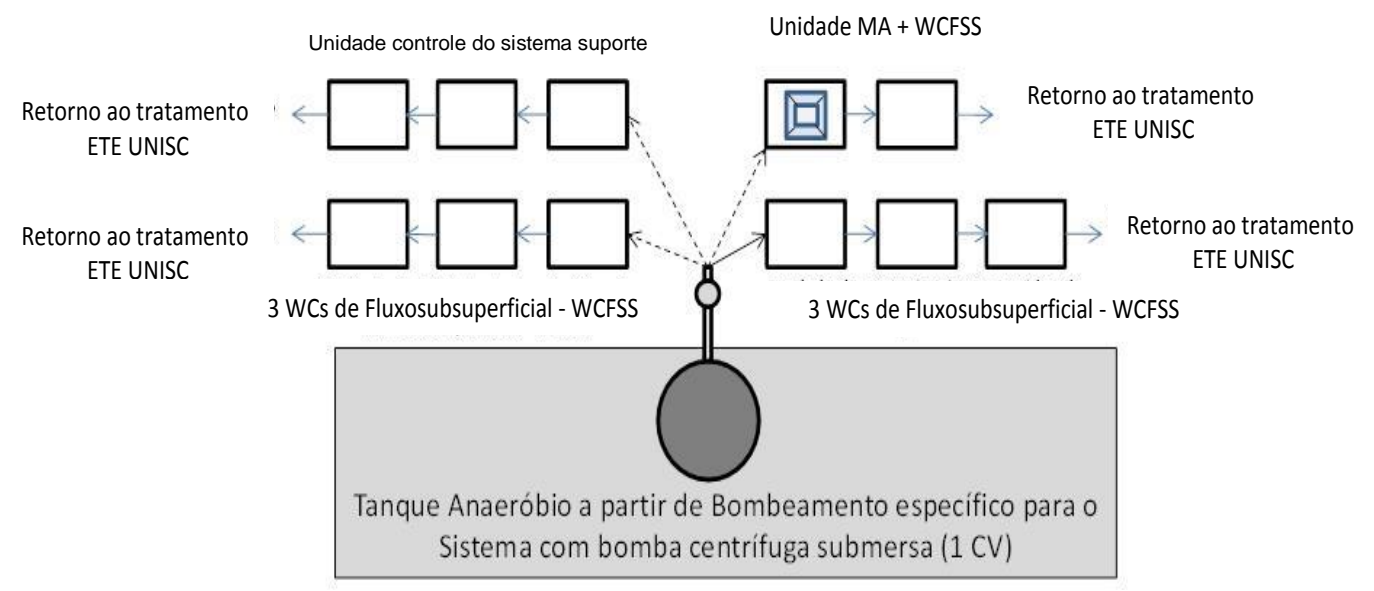

A segunda configuração ajustou a alimentação do sistema piloto MA + WCFV e CONTROLE, com linha própria de abastecimento, investindo em bomba submersa e painel de controle, criando assim, oportunidade de repetitividade da operação. 
Também foi eliminado o sistema de TQR da Figura 3. O detalhamento do sistema pode ser visto na Figura 5.

Figura 5 - Detalhe do sistema de MA sem recirculação externa em reator tipo coluna na CONFIGURAÇÃO 2.

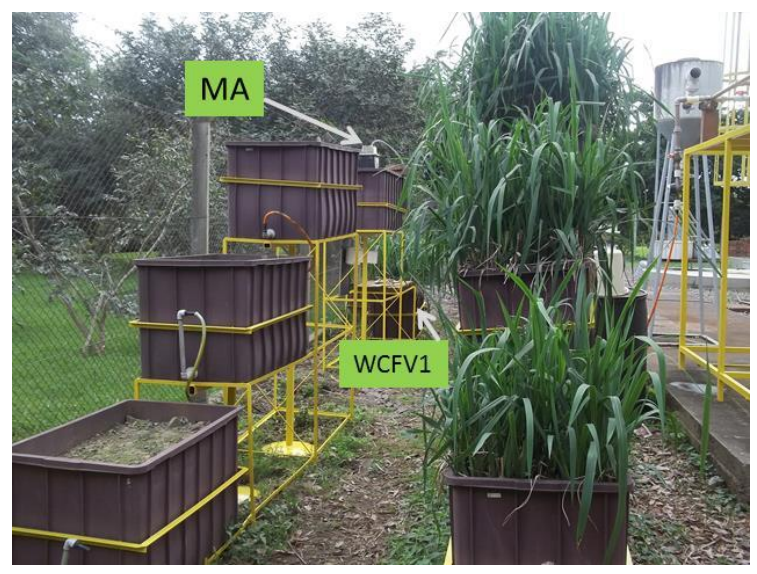

Com a CONFIGURAÇÃO 2 o problema remanescente foi para a quantidade de efluente a partir do tanque anaeróbio. Cada carga do sistema envolveu 30L para o MA e 90L para os demais sistemas, ou seja, a CONFIGURAÇÃO 2 ainda carecia de maior volume útil para o Reator Anaeróbio - RA. Assim foi feita a CONFIGURAÇÃO 3, evoluindo para o quinto e sexto mês de ensaios de operacionalidade da ETE piloto. Dificuldades operacionais semelhantes também foram enfrentadas por HORN, 2011. A configuração apresentada na Figura 6 também trouxe melhorias de segurança, sendo que as unidades de estrutura metálica foram reforçadas, impedindo riscos de quedas.

Figura 6 - CONFIGURAÇÃO 3 do sistema para início do controle sistemático da integração MA + WCFV (Vista superior).

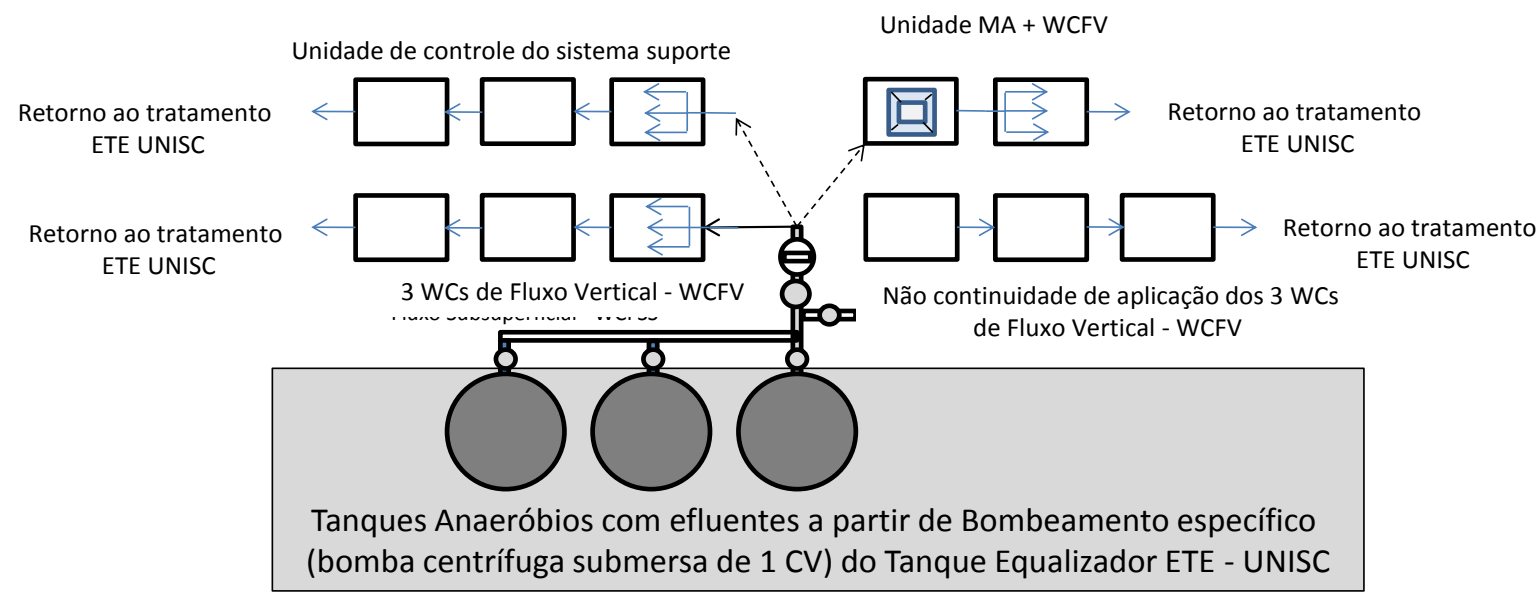


A CONFIGURAÇÃO 3 traz também as modificações definitivas três sistemas sequenciais, facilitando a operacionalidade de carga, bem como, aplicação do regime de alimentação vertical para os primeiros tanques dos WCs. Este sistema operou mais dois meses, fechando o ciclo dos seis primeiros meses de propósito das atividades. Na Figura 7 apresenta-se o detalhe do sistema de cargas do efluente. Evolução de operacionalidade também foi experimentada com os trabalhos de Silveira, 2010.

Figura 7 - Detalhe do sistema de RA interligado para alimentação das unidades MA e WCs.

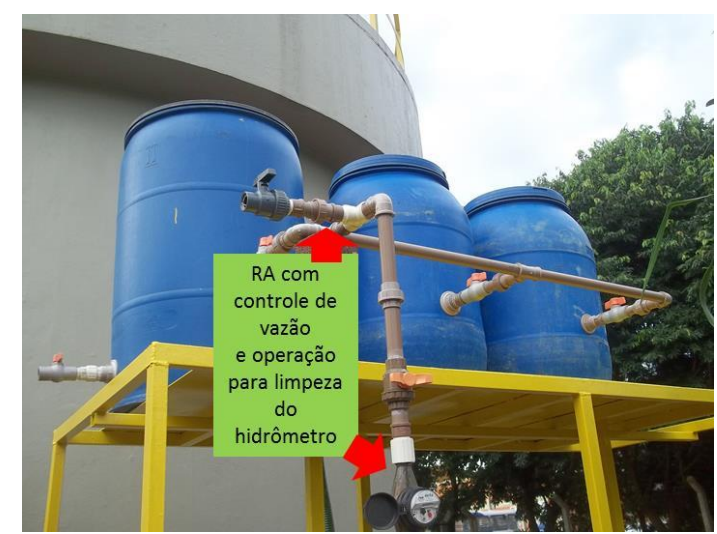

\subsection{Desempenho do sistema MA + WCFV1 - CONFIGURAÇÃO 3}

A sequência das Figuras de 8 a 10 demonstram os principais resultados de redução da carga poluente nos sistemas de tratamento em comparação.

Figura 8 - Sequência de resultados para variações dos parâmetros de carga poluente com depleção de oxigênio dissolvido.
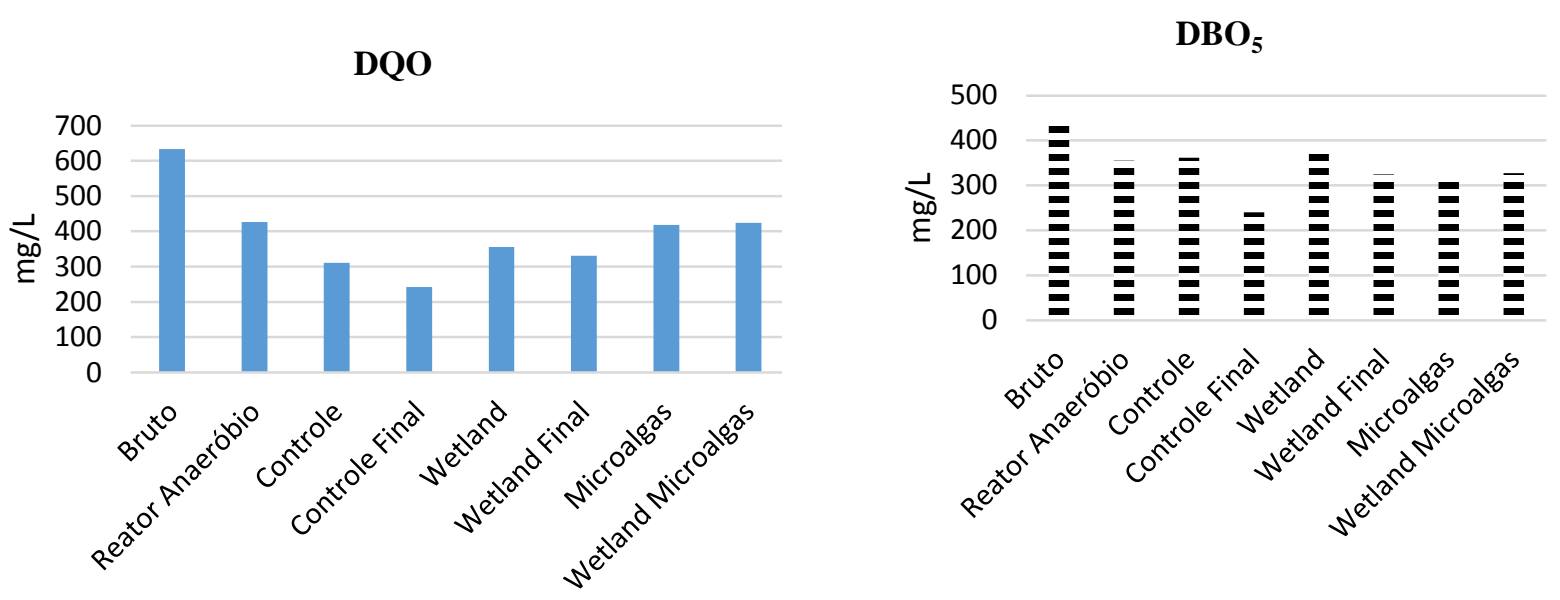
Considerando os resultados da Figura 8, pode ser observado que o desempenho da CONFIGURAÇÃO 3 necessita de melhorias quanto ao tempo de detenção, pois as reduções de $\mathrm{DQO}$ e $\mathrm{DBO}_{5}$ foram mínimas ou não observadas. Também deve ser referendado que o sistema de drenagem do reator anaeróbio necessitará de modificações, especialmente para melhor separação de fases lodo e efluente, bem como maior agitação ao longo da detenção para efetivar a anaerobiose.

No caso da ação de redução da carga eutrofizante, a remoção de fósforo foi ineficiente quando comparada aos WCs, mas efetiva quanto ao fator de carga, com $277,7 \mathrm{mg} \mathrm{P}$ total $\mathrm{cm}^{-1}$. Estes valores devem ser ajustados de acordo com o aplicado por Maddox e Kingsley, 1989.

Figura 9 - Sequência de resultados para variações dos parâmetros de carga poluente com ação eutrofizante.

\section{Fósforo Total}
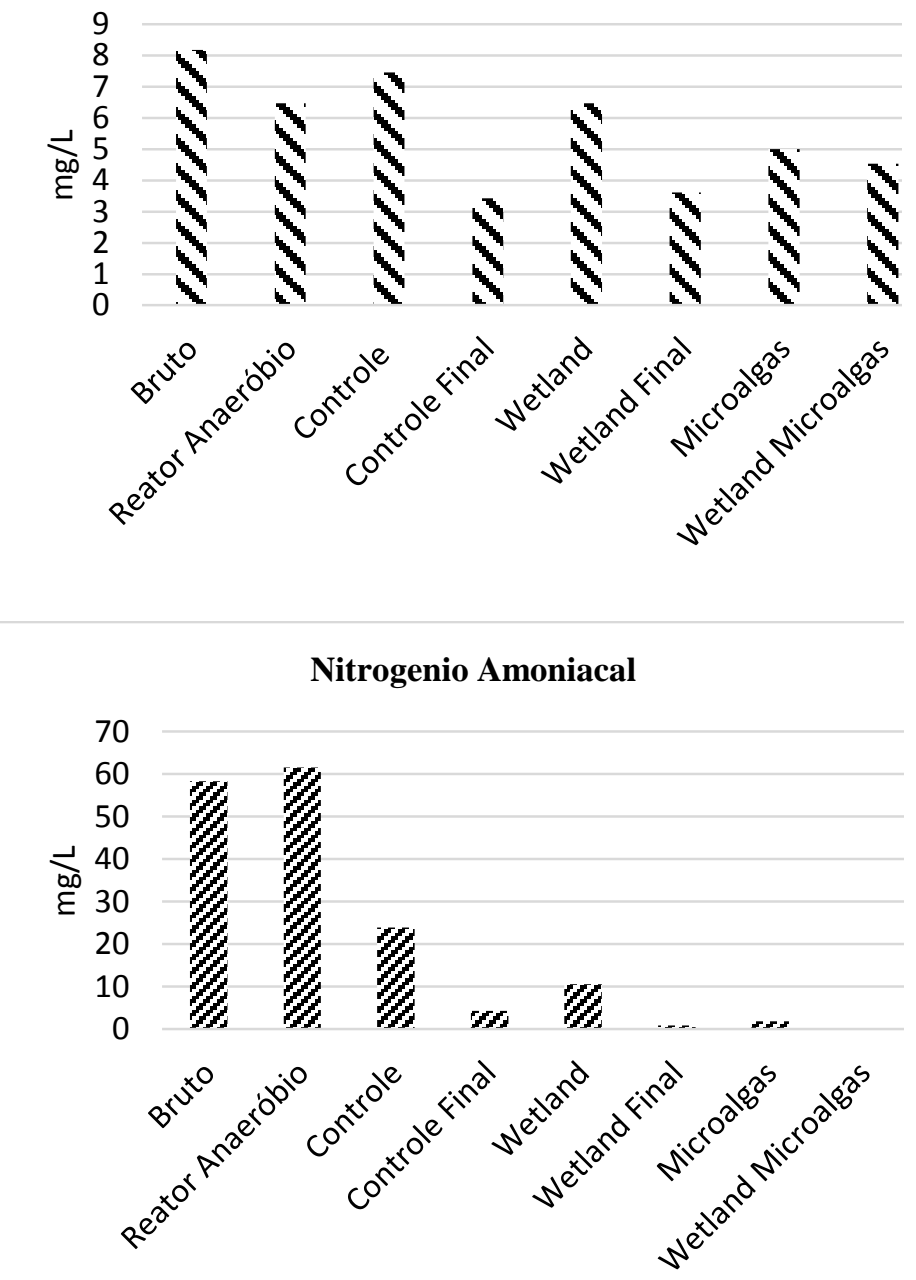

Revista Jovens Pesquisadores, Santa Cruz do Sul, v.6, n.2, p. 31-44, 2016. 
Já os dados de fitotoxicidade demonstraram ganhos ambientais significativos com o sistema MA e MA+WCFV, conforme observado na Figura 10, especialmente quanto a taxa de germinação. Isto está associado com a redução do $\mathrm{N}_{-} \mathrm{NH}_{4}{ }^{+}$, associação feita com os trabalhos de Dupont, 2010, onde a combinação das atividades de algas e sistema anóxico em MA foram os responsáveis.

Figura 10 - Sequência de resultados para variações dos parâmetros de carga poluente com ação de fitotoxicidade.
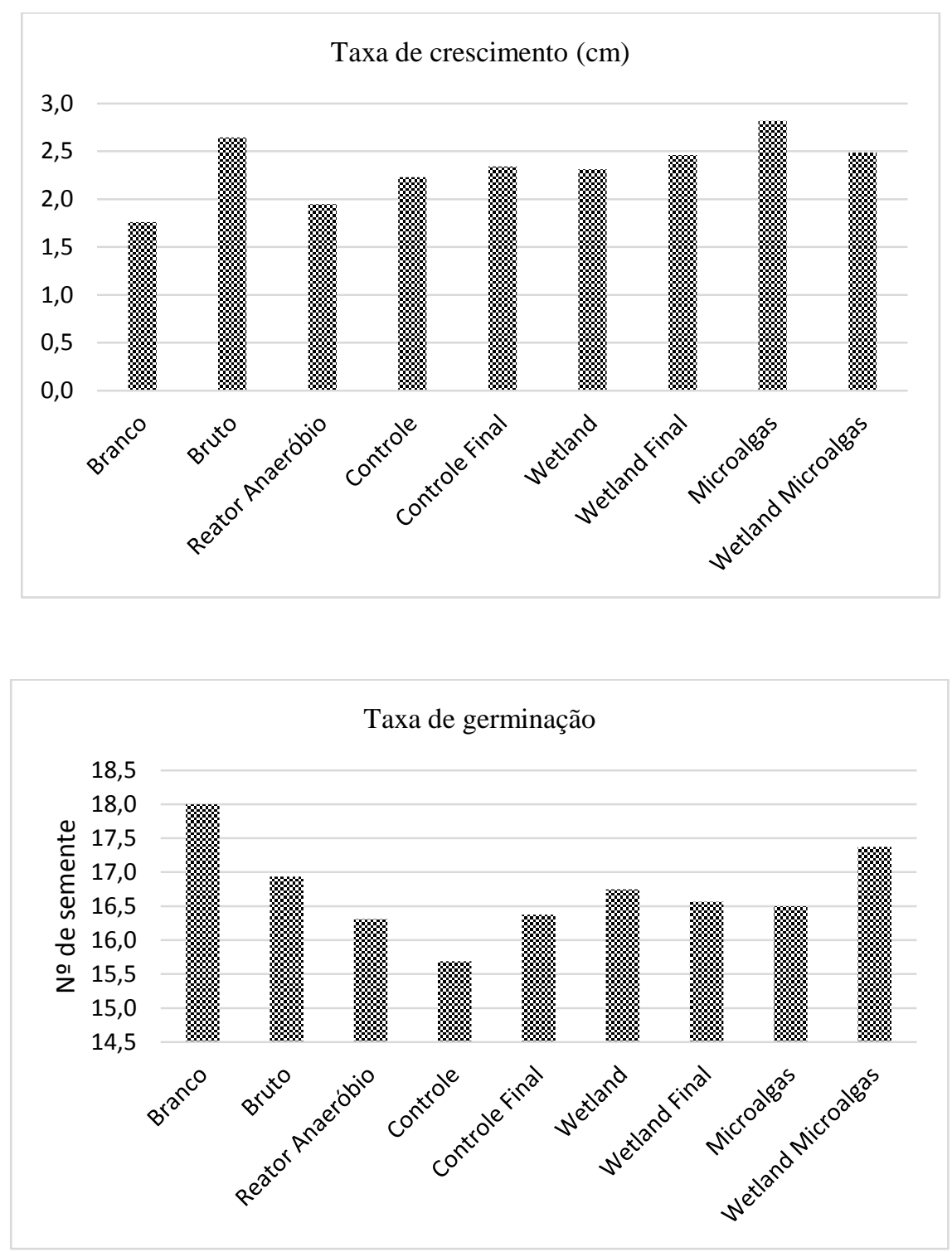

Entre os dados apresentados o que mais se destaca é a efetividade da redução de carga eutrofizante para o $\mathrm{N}_{-} \mathrm{NH}_{4}{ }^{+}$, especialmente porque o método de medida por via de titulação não mostrou interferências como o método ótico para determinar fósforo total. Neste 
caso, a turbidez residual não foi eliminada, o que provoca a interferência positiva.

Todos os parâmetros apresentaram uma redução na carga poluente comparando o efluente bruto com o pós-tratado, MA + WCFV, uma redução de 35\% de DQO, $22 \% \mathrm{DBO}_{5}$, $100 \%$ de nitrogênio amoniacal, $45 \%$ fósforo total, com uma taxa de germinação de $87 \%$. Porém, para o atendimento da resolução CONSEMA 128 (2006), melhorias deverão continuar como foco das pesquisas, especialmente para $\mathrm{DQO}, \mathrm{DBO}_{5}$ e fósforo total. Os demais dados de controle geral podem ser vistos na Tabela 1, reunindo a referência da avaliação preliminar da geração de microalgas no sistema MA.

Tabela 1 - Parâmetros de controle adicionais da CONFIGURAÇÃo 3.

\begin{tabular}{ccccc}
\hline & $\mathrm{pH}$ & Condutividade & Turbidez & Espectrofotometria \\
\hline Bruto & 7,65 & 1005,17 & 193,62 & 0,71 \\
\hline Reator Anaeróbio & 6,98 & 1107,09 & 50,14 & 0,21 \\
\hline Controle & 5,92 & 636,28 & 13,80 & 0,05 \\
\hline Controle Final & 6,28 & 618,01 & 0,82 & 0,03 \\
Wetland (WCFV1) & 5,72 & 528,88 & 11,53 & 0,24 \\
Wetland Final (WCFV2) & 6,50 & 677,43 & 2,77 & 0,08 \\
\hline Microalgas (MA) & 8,49 & 671,13 & 73,49 & 0,57 \\
Wetland Microalgas & & & 59,04 & 0,74 \\
(MA+WCFV) & 6,65 & 888,69 & $58 \mathrm{~nm}$. \\
\hline Leitura feita em 420nm, e para amostras contendo microalgas, 686
\end{tabular}

As reduções de turbidez e condutividade refletem a biomassa reduzida após o processo anaeróbio, especialmente para o WCFV1 e para o MA, indicando que melhor efetividade de fitorremediação no sistema WCFV2 poderá remover ação eutrofizante com fósforo total advindo do sistema coloidal das algas.

\section{CONSIDERAÇÕES FINAIS}

O melhor desenho para operação de desempenho do sistema MA + WCFV 1 foi obtida com a chamada CONFIGURAÇÃO 3, agregando melhor controle para reprodutibilidade dos dados e segurança operacional. Especialmente o reator MA está adequado para reprodução dos dados em escala real, pois está simplificado para distribuição dos efluentes em filmes capazes de permitir em vazões de $0,1 \mathrm{Lmin}^{-1} \mathrm{~m}^{-2}$, a taxa de 
desenvolvimento das algas em tempos de detenção de 3 dias.

Reduções totais de $\mathrm{N}^{-\mathrm{NH}_{4}}{ }^{+}$revelam que o sistema MA + WCFV1 tem potencial para controle da eutrofização, sendo que para o controle do agente eutrofizante mais crítico, o fósforo, melhorias de controle operacional e analítico devem ser feitas, especialmente quanto aos parâmetros de carga e também no controle da interferência dos colóides no método ótico.

\section{AGRADECIMENTOS}

Agradecimentos a UNISC e ao CNPq com o financiamento para o projeto número 481620/2013-3.

\section{REFERÊNCIAS}

APHA/AWWA - AMERICAN PUBLIC HEALTH ASSOCIATION. Standard Methods for the Examination of Water and Wastewater. 21. ed. Washington: APHA/AWWA/WEF,2005.

CONSEMA - CONSELHO ESTADUAL DE MEIO AMBIENTE - Resolução n. 128/2006.

Disponível em: <http://www.sema.rs.gov.br/upload/Resolucao\%20CONSEMA\%202452010.pdf >. Acesso em: junho de 2014.

DUFRESNE, K. et al. Metal Retention Mechanisms in Pilot-Scale Constructed Wetlands Receiving Acid Mine Drainage, 10 th International Conference on Acid Rock Drainage and IMWA Annual Conference, p.1-6, 2015.

DUPONT, A. Avaliação da eficiência da Estação de Tratamento de Esgoto da Universidade de Santa Cruz do Sul, RS, Brasil. 2010. 159 f. Dissertação (Programa de Pós-Graduação em Tecnologia Ambiental - Mestrado e Doutorado) - Universidade de Santa Cruz do Sul, Santa Cruz do Sul, 2010.

HERRERA-MELIÁN, J.A. et al. Degradation and detoxification of 4-nitrophenol by advanced oxidation technologies and bench-scale constructed wetlands. Journal of Environmental Management,105, p.53-60, 2012.

HORN, T. B. Integração de sistemas wetlands construídos + fotoozonização catalítica no tratamento de efluentes de campus universitário. 2011. 157 f. Dissertação (Programa de Pósgraduação em Tecnologia Ambiental- Mestrado e Doutorado) - Universidade de Santa Cruz do Sul, Santa Cruz do Sul, 2011.

LU, S.; PEI, L.; BAI, X. Study on method of domestic wastewater treatment through newtype multi-layer artificial wetland. International Journal of Hydrogen Energy, v. 40 (34), p. 11207- 11214, 2015.

MADDOX, J. J.; KINGSLEY, J. B. Waste treatment for confined swine with na integrated artificial wetland and aquaculture system, Chapter 14. In: HAMMER, D. A. Constructed etlands for wastewater treatment: municipal, industrial and agricultural, CRC Press, 628 págs. 1989. 
PHILIPPI, L. S.; SEZERINO, P. H. Aplicação de sistemas tipo wetlands no tratamento de águas residuárias: utilização de filtros plantados com macrófitas. Florianópolis: Editora do Autor, 2004.

RAKOCZY, J. et al. A Bench-Scale Constructed Wetland As a Model to Characterize Benzene Biodegradation Processes in Freshwater Wetlands. Environ. Sci. Technol., 45 (23), pp 10036-10044, 2011.

SEZERINO, P. H. Potencialidade dos filtros plantados com macrófitas (constructed wetlands) no pós-tratamento de lagoas de estabilização sob condições de clima subtropical. 2006. 171 f. Tese (Programa de Pós-Graduação em Engenharia Ambiental - Mestrado e Doutorado) - Universidade Federal de Santa Catarina, Florianópolis, 2006.

SILVEIRA, D. D. Estudos Fenológicos da macrófita Hymenachne grumosa (MagnoliophytaPoaceae) na aplicação de Wetlands construídos para o tratamento de efluentes secundários de campus universitário. 2010. 103 f. Dissertação (Programa de Pós-graduação em Tecnologia Ambiental- Mestrado e Doutorado) - Universidade de Santa Cruz do Sul, Santa Cruz do Sul, 2010.

SILVEIRA, E. O. et al. Sistema integrado de tratamento de efluentes urbanos com microalgas e wetlands construídos. In: Segundo Simpósio Brasileiro Sobre Wetlands Construídos. Curitiba: UTFPR, v. 2. p. 1-10, 2015.

VAN KAICK, T. S. Estação de tratamento de esgoto por meio de zona de raízes: uma proposta de tecnologia apropriada para saneamento básico no litoral do Paraná. 2002. 128 f. Dissertação (Mestrado em Tecnologia) - Universidade Tecnológica Federal do Paraná, Curitiba, 2002.

ZERWES, F. V. Sistema de tratamento e reúso de águas residuárias em Pequenas propriedades rurais: acompanhamento e operação de Estação no vale do rio pardo - RS. 2013. 69 f. Dissertação (Programa de Pós-Graduação em Tecnologia Ambiental - Mestrado e Doutorado) - Universidade de Santa Cruz do Sul, 2013.

ZERWES, F. V. et al. Avaliação citogenética do tratamento com o processo uasb/biofiltro anaeróbio/wetlands construídos de fluxo subsuperficial (WCFSS) de efluentes de propriedade rural. Revista Engenharia e Construção Civil, v. 2 (1), p. 52-60, 2015.

\section{Como citar este documento:}

WINK, Matheus et al. Unidade piloto em regime de batelada com sistema de reatores anaeróbios + microalgas + wetlands construídos em fluxo vertical. Revista Jovens Pesquisadores, Santa Cruz do Sul, v. 6, n. 2, nov. 2016. ISSN 2237-048X. Disponível em: 〈https://online.unisc.br/seer/index.php/jovenspesquisadores/article/view/7303 >. Acesso em: ... doi:http://dx.doi.org/10.17058/rjp.v6i2.7303. 